\title{
Comprehensive pharmacogenomic characterization of gastric cancer
}

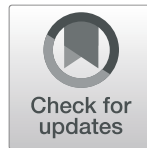

\begin{abstract}
Jason K. Sa ${ }^{1+}$ D, Jung Yong Hong ${ }^{2 \dagger}$, In-Kyoung Lee ${ }^{2}$, Ju-sun $\mathrm{Kim}^{2}$, Moon-Hee Sim², Ha Jung Kim², Ji Yeong An ${ }^{3}$, Tae Sung Sohn³, Joon Ho Lee ${ }^{3}$, Jae Moon Bae ${ }^{3}$, Sung Kim³, Kyoung-Mee Kim, Seung Tae Kim², Se Hoon Park², Joon Oh Park ${ }^{2}$, Ho Yeong Lim², Won Ki Kang ${ }^{2}$, Nam-Gu Her ${ }^{5}$, Yeri Lee ${ }^{5}$, Hee Jin Cho ${ }^{5}$, Yong Jae Shin ${ }^{5}$, Misuk Kim ${ }^{5}$, Harim Koo ${ }^{5,6}$, Mirinae Kim ${ }^{5}$, Yun Jee Seo ${ }^{5}$, Ja Yeon Kim ${ }^{5}$, Min-Gew Choi ${ }^{3^{*}}$, Do-Hyun Nam,6,7* and Jeeyun Lee ${ }^{2^{*}}$
\end{abstract}

\begin{abstract}
Background: Gastric cancer is among the most lethal human malignancies. Previous studies have identified molecular aberrations that constitute dynamic biological networks and genomic complexities of gastric tumors. However, the clinical translation of molecular-guided targeted therapy is hampered by challenges. Notably, solid tumors often harbor multiple genetic alterations, complicating the development of effective treatments.

Methods: To address such challenges, we established a comprehensive dataset of molecularly annotated patient derivatives coupled with pharmacological profiles for 60 targeted agents to explore dynamic pharmacogenomic interactions in gastric cancers.

Results: We identified lineage-specific drug sensitivities based on histopathological and molecular subclassification, including substantial sensitivities toward VEGFR and EGFR inhibition therapies in diffuse- and signet ring-type gastric tumors, respectively. We identified potential therapeutic opportunities for WNT pathway inhibitors in ALK-mutant tumors, a significant association between PIK3CA-E542K mutation and AZD5363 response, and transcriptome expression of RNF11 as a potential predictor of response to gefitinib.
\end{abstract}

Conclusions: Collectively, our results demonstrate the feasibility of drug screening combined with tumor molecular characterization to facilitate personalized therapeutic regimens for gastric tumors.

Keywords: Gastric cancer, Pharmacogenomics, PIK3CA-E542K, RNF11, Gefitinib

\section{Background}

Cancer is a complex disease, with profound genomic alterations and diverse cellular hierarchy [1-4]. Advancements in the field of genetics have enabled us to achieve a comprehensive understanding of the tumor molecular structure and the impacts of core oncogenic pathways that are frequently dysregulated [3, 5-7]. However, the development of effective treatments based on molecular

\footnotetext{
*Correspondence: mingew.choi@samsung.com; nsnam@skku.edu; jyunlee@skku.edu

${ }^{\dagger} J a s o n$ K. Sa and Jung Yong Hong contributed equally to this work. ${ }^{3}$ Department of Surgery, Samsung Medical Center, Sungkyunkwan University School of Medicine, Seoul, Republic of Korea

${ }^{5}$ Institute for Refractory Cancer Research, Samsung Medical Center, Seoul, Republic of Korea

${ }^{2}$ Division of Hematology-Oncology, Department of Medicine, Samsung Medical Center, Sungkyunkwan University School of Medicine, Seoul, Republic of Korea

Full list of author information is available at the end of the article
}

characterization of the tumor alone has been increasingly recognized to be limited due to the co-existence of multiple genomic aberrations within a given tumor. To address this challenge, large-scale drug sensitivity profiles of conventional cancer cell-line models have been employed to identify clinically relevant biomarkers that could be therapeutically exploited [8-11]. While these studies have provided unprecedented insights into the biological signaling networks that govern dynamic cellular responses to a broad range of therapeutics, the heterogeneous biological traits of patient-derived tumors hamper the direct application of the current pharmacogenomic atlas in the clinic. We have previously established a compilation of chemical-genetic associations across a wide spectrum of patient-derived tumor cell (PDC) models and demonstrated its clinical feasibility $[12,13]$. To further interrogate the dynamics of pharmacogenomic interactions at

(c) The Author(s). 2020 Open Access This article is distributed under the terms of the Creative Commons Attribution 4.0 International License (http://creativecommons.org/licenses/by/4.0/), which permits unrestricted use, distribution, and reproduction in any medium, provided you give appropriate credit to the original author(s) and the source, provide a link to the Creative Commons license, and indicate if changes were made. The Creative Commons Public Domain Dedication waiver (http://creativecommons.org/publicdomain/zero/1.0/) applies to the data made available in this article, unless otherwise stated. 
a single tumor-lineage resolution, we established a library of tumor cell models from surgically resected tumor specimens or ascites-derived tumor cells from gastric cancers and explored potential gene-drug associations for 60 molecularly targeted agents.

Gastric cancer is the third leading cause of cancerinduced mortality worldwide [14-16]. The vast majority of gastric tumors are diagnosed as adenocarcinomas and can be subcategorized into distinct classifications based on molecular, histological, and pathological features [3, 17]. The current standard regimen consists of surgical resection followed by radio-chemotherapy. Although integrated molecular characterization of gastric adenocarcinomas through nationwide efforts of The Cancer Genome Atlas Research Network has led to the identification of major genetic aberrations and oncogenic pathways that contribute to the malignancy of gastric cancer, the clinical application potential of molecular targeted therapy remains obscure. To date, only two molecular target agents, including trastuzumab (anti-HER2 monoclonal antibody) tested in the ToGA trial and ramucirumab (anti-VEGFR monoclonal antibody) investigated in the RAINBOW and REGARD trials have been approved for clinical treatment of metastatic gastric cancer [18-20]. Numerous clinical trials on agents targeting major oncogenic pathways, including HER2 (lapatinib, pertuzumab, trastuzumab-emtansine), EGFR (cetuximab, panitumumab, nimotuzumab, gefitinib), FGFR2 (AZD4547), VEGF (bevacizumab, aflibercept), MET (onartuzumab, rilotuzu$\mathrm{mab}$ ), and PI3K/AKT/mTOR (PAM; ipatasertib, everolimus) have shown disappointing results despite promising preclinical evidences [16]. The current limitations in applicability of molecular-guided therapy are presumably due to inadequate patient stratification and the extensive inter-tumoral heterogeneity of gastric tumors. To address these challenges, we analyzed somatic mutations, copy number alterations, and/or gene expression profiles of 131 gastric tumors as potential predictors of drug sensitivities for 60 anti-cancer compounds to identify molecular determinants that may aid in a paradigm shift towards personalized treatment of gastric cancer.

\section{Methods}

\section{Gastric tumor specimens and in vitro cell culture}

After receiving informed consent, gastric tumor specimens or malignant ascites were obtained from patients undergoing surgery at Samsung Medical Center (SMC) in accordance with the Samsung Medical Center Institutional Review Board. This study was conducted in compliance with all relevant ethical regulations for human specimen research. Portions of the surgical samples were enzymatically dissociated using Liberase ${ }^{\text {Tw }}$ (Roche) and tumor cells from malignant effusions were collected by centrifugation at $300 \mathrm{~g}$ for $10 \mathrm{~min}$, followed by washing with Dulbecco's phosphate-buffered saline. Patient-derived tumor cells (PDCs) were cultured in neurobasal medium with $\mathrm{N} 2$ and B27 supplements $(0.5 \times$ each; Thermo Fisher Scientific) and human recombinant basic fibroblast growth factor and epidermal growth factor $(20 \mathrm{ng} / \mathrm{ml}$; R\&D Systems). Human gastric cancer cell-lines were purchased from the Korean Cell Line Bank. All cell lines were cultured in RPMI 1640 medium supplemented with $10 \%$ fetal bovine serum and Antibiotic-Antimycotic (penicillin and streptomycin; Invitrogen) at $37{ }^{\circ} \mathrm{C}$ in a humidified atmosphere with $5 \% \mathrm{CO}_{2}$. PDCs and all cancer cell-lines were tested for mycoplasma contamination.

\section{Exome sequencing}

Tumors were subjected to target exome sequencing using CancerSCAN, a targeted sequencing platform designed at Samsung Medical Center. CancerSCAN covers a range of exonic regions of specific genes that are associated with cancer progression. Genomic DNA was sheared in Covaris S220 sonicator (Covaris) to construct a sequencing library using the SureSelect XT Reagent Kit, HSQ (Agilent Technologies), enriched for target genes. The library was purified and amplified with a barcode tag, and library quality and quantity were determined. Sequencing was carried out using the 100-bp paired-end mode of the TruSeq Rapid PE Cluster kit and TruSeq Rapid SBS kit on a HiSeq 2500 sequencing platform (Illumina). The target exome sequencing data of previous gastric cancer cases were downloaded from the European Genome-phenome Archive (EGAS00001002515).

\section{Mutation calls}

The sequenced reads in FASTQ files were aligned to the human genome assembly (hg19) using the BurrowsWheeler Aligner. The initial alignment BAM files were subjected to sorting (SAMtools), removal of duplicated read (Picard), local realignment of reads around potential small insertions/deletions, and recalibration of the base quality score (Genome Analysis Toolkit). MuTect was used to generate high-confidence mutation calls. Variant Effector Predictor was used to annotate the called mutations.

\section{Copy number alteration}

ONCOCNV was used to generate estimated copy number alterations in tumor specimens.

\section{RNA sequencing}

RNA-seq libraries were prepared using the Illumina TruSeq RNA Sample Prep kit. Sequenced reads were mapped onto hg19 using the Burrows-Wheeler Aligner. The initial BAM files were sorted and summarized into BED files using SAMtools and bedTools. The BED files were used to calculate the reads per kilobase of transcript per million 
reads (RPKM) value for each gene, using the DEGseq package.

\section{Drug screening}

PDCs were cultured in serum-free medium, dissociated into single cells, and seeded in 384-well plates at 500 cells/ well in duplicate or triplicate for each treatment. The drug panel consisted of 60 molecular target agents targeting oncogenic signals (Additional file 1: Table S1). All drugs were purchased from Selleckchem. PDCs were treated with the drugs in a fourfold or seven-point serial dilution series from $4.88 \mathrm{nM}$ to $20 \mu \mathrm{M}$ using Janus Automated Workstation (PerkinElmer). After 7 days of incubation, cell viability was analyzed using ATP monitoring system based on firefly luciferase (ATPLite ${ }^{\mathrm{Tm}} 1$ step; PerkinElmer). Viable cells were estimated using EnVision Multilabel Reader (PerkinElmer). Control cells treated with dimethyl sulfoxide (DMSO) vehicle were used to calculate relative cell viability for each plate and to normalize the data on a per-plate basis. Dose response curve fitting was performed using GraphPad Prism 5 (GraphPad) and was evaluated by measuring the area under curve (AUC). In brief, each plate was normalized to the mean value from the seven serial conditions compared with DMSO control. The AUC of each curve was determined using GraphPad Prism (GraphPad Software), ignoring regions defined by fewer than two peaks. Non-convergence or ambiguous curves were excluded in every analysis. Two identical PDCs and cancer cell-lines were screened every month to validate and confirm the preservation of chemical activities of our drug library and high-throughput drug screening platform. Screening plates were subjected to quality control measurement using $z$-factor scores, comparing both negative and positive control wells [21].

\section{Pharmacogenomic interactions of genetic variations}

A list of genetic variations, including single nucleotide variations, small insertions, small deletions, and copy number alterations, were considered to evaluate drug response interactions. For each drug candidate, drug sensitivity data (AUC) were analyzed by comparing tumors with the selected genomic alterations to those without using the Wilcoxon rank-sum test. Samples with unknown status of a given alteration were excluded from the analysis.

\section{Elastic net regression model-based analysis}

We selected 41 gastric cancer cases with available RNAseq and drug response data. The input variables for the elastic net regression model-based analysis consisted of gene expression profiles with genomic alterations including mutations and copy number alterations for tumors whose genome data were available. We then trained the standard elastic net regression using the glmnet $\mathrm{R}$ package by combining input features and comparing to individual drugs response. Afterwards, we employed bootstrapping strategy for 100 times to extract reliable and robust candidate features. During each bootstrapping step, we randomly selected $80 \%$ of the tumors for feature extraction. For each feature, the time of its appearance out of 100 bootstrapping and its average weight were used as the final assessment.

\section{Cell establishment and growth assessment against gefitinib}

Gastric cancer cell-lines were transiently transfected with $10 \mathrm{nM}$ of siRNF11 using $6 \mu \mathrm{L}$ of HiPerFect transfection reagent (Qiagen). Next day, the cells were seeded in 96-well plates at 5000 cells/well, allowed to adhere overnight, and treated with gefitinib for $72 \mathrm{~h}$. Cell proliferation inhibition was determined using CellTiter-Glo Luminescent Cell Viability Assay (Promega) according to the manufacturer's protocol. The detected luminescent signals were used to calculate the percentage of surviving cells and to obtain AUC values.

\section{Cellular growth assessment against AZD5363}

Gastric cancer cell-lines with PIK3CA mutation (E542K or E545K) or wild-type have been seeded in 96-well plates at 5000 cells per well, allowed to adhere overnight and treated with various concentrations $(0.3$ or $1 \mu \mathrm{M})$ of AZD5363 for $72 \mathrm{~h}$, and the cell viability was determined using the Cell Titer Glo.

\section{siRNA sequences}

siRNA constructs for RNF11 (siRNF11\#1: 5'-ACATCT CCCTGCTTCACGAC-3' and siRNF11\#2: 5'-GGAAGA GAUGGAUCAGAAA-3') and control (siControl: 5' TAGCGACTAAACACATCAA-3') were used in this study.

\section{Immunoblot analysis}

Total cell extracts were prepared using lysis buffer $(20 \mathrm{mM}$ HEPES [pH 7.4], 1\% Triton X-100, $1 \mathrm{mM}$ EDTA, $1 \mathrm{mM}$ $\mathrm{MgCl}_{2}, 150 \mathrm{mM} \mathrm{NaCl}, 10 \%$ glycerol, protease inhibitor, and phosphatase inhibitor cocktail [Invitrogen]). Protein concentrations were determined using micro-BCA protein reagent (Pierce Biotechnology). Thirty micrograms of total proteins were separated by sodium dodecyl sulfate polyacrylamide gel electrophoresis and transferred onto nitrocellulose membranes with $0.2-\mu \mathrm{m}$ pore size (Whatman). The membranes were incubated with antibodies against phospho-AKT (Ser473) (\#4060, 1:1000; Cell Signaling Technology (CST); RRID: AB_2315049), AKT (\#9272, 1:1000; CST; RRID: AB_329827), phospho-mTOR (Ser2448) (\#2971, 1:1000; CST; RRID: AB_330970), mTOR (\#2972, 1:1000; CST; RRID: AB_330978), phospho-S6 ribosomal protein (Ser2235/236) (\#2211, 1:1000; CST; RRID: AB_331679), S6 ribosomal protein (\#2217, 1:1000; CST; 
RRID: AB_331355), phospho-4E-BP1 (Thr70) (\#13396, 1: 1000; CST; RRID: AB_2798206), 4E-BP1 (\#9644, 1:1000; CST; RRID: AB_2097841), phospho-EGFR (Tyr1068) (\#3777, 1:1000; CST; RRID: AB_2096270), EGFR (\#2646, 1:1000; CST; RRID: AB_2230881), RNF11 (ab154831, 1: 1000; Abcam), or $\beta$-actin (AC-15, 1:5000; Sigma; RRID: AB_476692). The ECL method (Invitrogen) was used for protein detection.

\section{Statistical analysis}

Data are presented as the mean \pm standard deviation (SD). All statistical analyses were conducted by either Wilcoxon rank-sum test (two-sided), Pearson's correlation coefficient test, or Fisher's exact test (two-sided) as relevant. All statistical analyses were conducted using the $\mathrm{R}$ software (https://www.r-project.org) or GraphPad Prism.

\section{Results}

\section{Mutational landscape of gastric cancer}

To explore the dynamics of pharmacogenomic interactions in gastric cancers, we generated 131 surgically resected gastric tumor specimens or malignant ascites (Additional file 2: Table S2). To determine genomic variations, including single-nucleotide variants (SNVs), small insertions/deletions (Indels), and copy number alterations (CNAs; segments of the genome that are either amplified or deleted), 102 tumor specimens were subjected to targeted massively parallel sequencing, covering the full coding exons of major cancer-driver genes (Additional file 3: Table S3). Mutations with variant allele frequency of $>5 \%$ and $>20$ reads were considered (Additional file 4: Table S4). Forty-one tumors were subjected to whole-transcriptome sequencing to curate gene expression profiles. Tumors were classified into four subgroups based on molecular profiles: Epstein-Barr Virus (EBV)-positive, microsatellite instability (MSI)-high, high copy number alterations (HCNA), and low copy number alterations (LCNA) (Additional file 5: Figure S1). HCNA tumors exhibited enrichment of TP53 mutation, whereas LCNA and EBV-positive tumors were marked by high prevalence of $C D H 1$ and $P I K 3 C A$ mutations, respectively (Fig. 1a, b) [3, 22]. Notably, LCNA tumors showed recurrent genetic aberrations of NF1, suggesting potential therapeutic opportunities for RAS/MAPK-targeted therapies [23]. Furthermore, the HCNA group demonstrated a significantly higher rate of HER2-positive tumors than the other types. Compared with other large-scale gastric cancer datasets, our cohort constituted comparable levels of major gastric cancer-driver genes, including somatic mutations of TP53, ARID1A, PIK3CA, and APC (Additional file 5: Figure S2) [3, 24, 25]. On note, our cohort harbored higher frequency of $C D H 1$-mutant tumors and we suspect that this could be due to the higher number of LCNA or genomically stable tumors, which are marked by enrichment of $C D H 1$ mutation (Additional file 5: Figure S2).

Compared with traditional long term-cultured cancer cell-line models, PDCs recapitulate the molecular properties and biology of diseases more precisely, prompting their feasibility as a reliable model system for evaluating the potential clinical response to various therapeutics [26-28]. One hundred twenty-eight gastric PDCs were cultured under serum-free conditions and were used in a systematic drug sensitivity screening of 60 compounds that target major oncogenic pathways, including receptor tyrosine kinases (RTKs), poly(ADP-ribose) polymerase (PARP), and histone deacetylase (HDAC) (Additional file 1: Table S1 and Additional file 5: Figure S3A). Drug sensitivities were determined based on the area under curve (AUC) of the dose-response curve after 7 days of treatment (Additional file 6: Table S5). For most compounds, the PDCs exhibited a wide range of sensitivities. Interestingly, t-stochastic neighbor embedding (tSNE) analysis revealed a hierarchical clustering of the RTK inhibitors, confirming the validity of our systematic screening procedure and the target inhibitor quality assessment (Additional file 5: Figure S3B). A number of PDC lines were further subjected to exome sequencing to interrogate whether they retained the spectrum of genomic aberrations observed in the matched tumor specimens. Consistent with previous findings, major cancer-driver alterations, including TP53, CDH1, PIK3CA, ERBB3, and FGFR3 mutations were highly preserved in the PDCs (Fig. 1c) [12, 13]. Collectively, these results suggest that PDCs recapitulate tumor molecular properties and can serve as proxies for comprehensive pharmacogenomic analyses in gastric tumors.

\section{Subgroup-specific drug sensitivities based on molecular, histological, and pathological classifications}

As gastric cancers can be subcategorized based on molecular, histological, and pathological features, we evaluated the pharmacological landscape of distinct subgroups of gastric PDCs within each subtype category. Overall, we observed a wide range of drug sensitivities within each class, demonstrating the highly heterogeneous nature of gastric PDCs (Additional file 5: Figure S4A and Additional file 7: Table S6). Molecular variations across each subcategory potentially contribute to dynamic drug responses. Of note, diffusesubtype tumors were highly sensitive to multiple RTK inhibitors targeting VEGFR, PDGFR, and FGFR pathways (cediranib, vandetanib, pazopanib, regorafenib, AZD4547, and BGJ398), whereas mixed types were mostly resistant (Fig. 2a). Furthermore, PDCs from signet-ring type demonstrated considerable sensitivities to EGFR inhibitors, including AEE788, afatinib, dacomitinib, and gefitinib, which were comparatively 


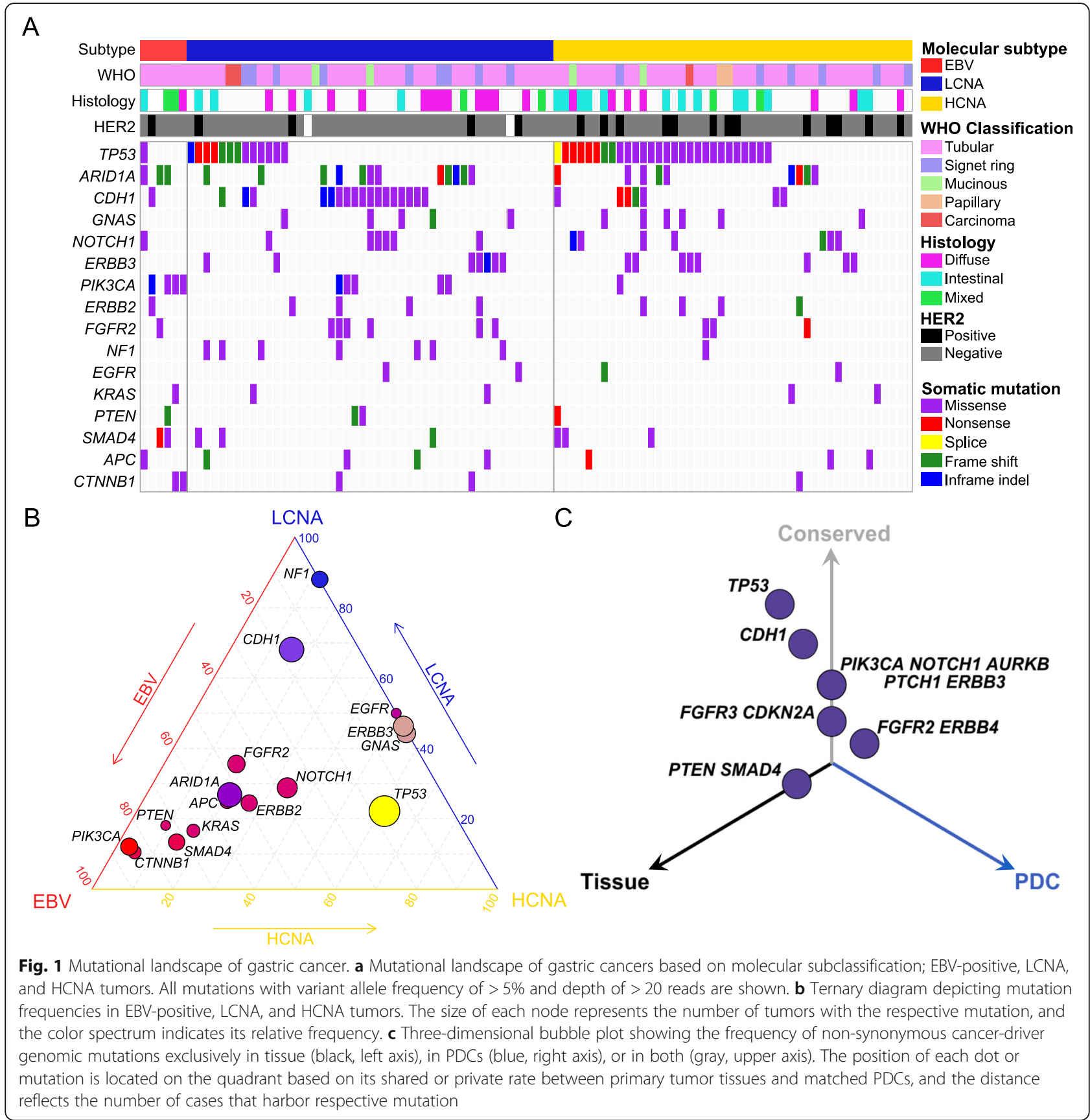

less potent in tubular subtype. HER2-positive tumors were highly sensitive to PAM compounds, including BEZ235 and PF-05212384, when compared with HER2-negative tumors. Consistent with the heterogeneous pharmacological behaviors of gastric PDCs, pathway enrichment analysis revealed enrichments of the EGFR, VEGFR, and ERBB2-PI3K pathways in signetring, diffuse, and HER2-positive tumors, respectively (Fig. 2b). Moreover, tumors with high chromosomal instability were considerably more sensitive to olaparib.
PARP inhibition therapy has demonstrated significant therapeutic success in patients diagnosed with either advanced ovarian or metastatic breast cancer with germline BRCA1/2 mutations $[29,30]$. Therefore, we sought to evaluate the prevalence of $B R C A 1 / 2$ mutations in each molecular gastric tumor subtype. Notably, BRCA2 mutation constituted a significant proportion in HCNA tumors, suggesting clinical application potential of olaparib for patients with high chromosomal instability and $B R C A 2$ variation (Additional file 5: Figure S4B). Furthermore, we 


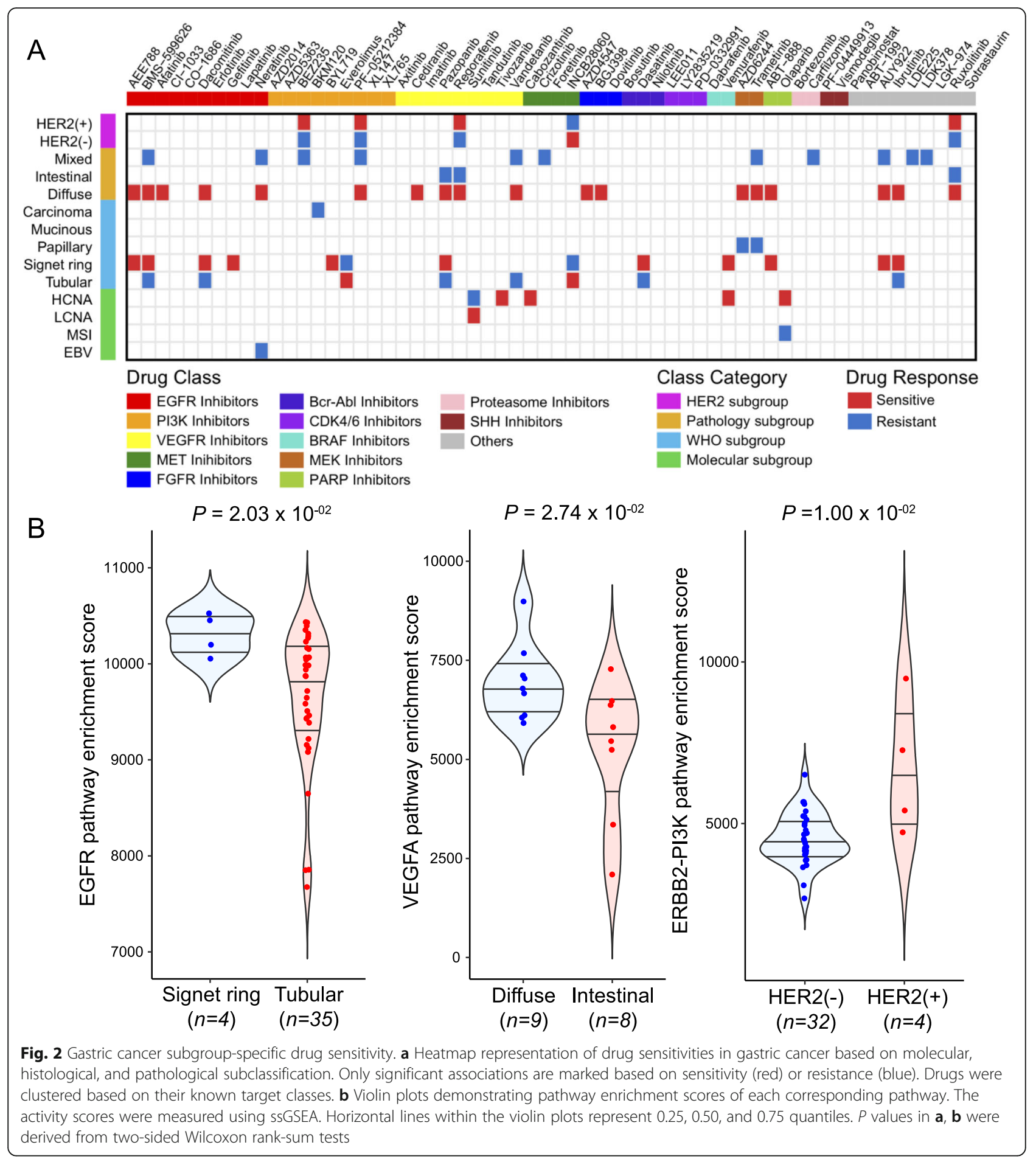

found that majority of the BRCA2-mutant tumors were $C D H 1$ wild-type (Additional file 5: Figure S4B). Together, these results underscore the significance of systematic drug sensitivity screening to guide subtypespecific targeted therapeutic opportunities in gastric cancers.
Pharmacogenomic landscape of gastric cancer

Genomic variations, including somatic mutations and copy number alterations, can be employed as reliable biomarkers for predicting clinical response to targeted therapy [31-34]. To identify genomic correlates of pharmacological sensitivity in gastric tumors, we evaluated individual drug 


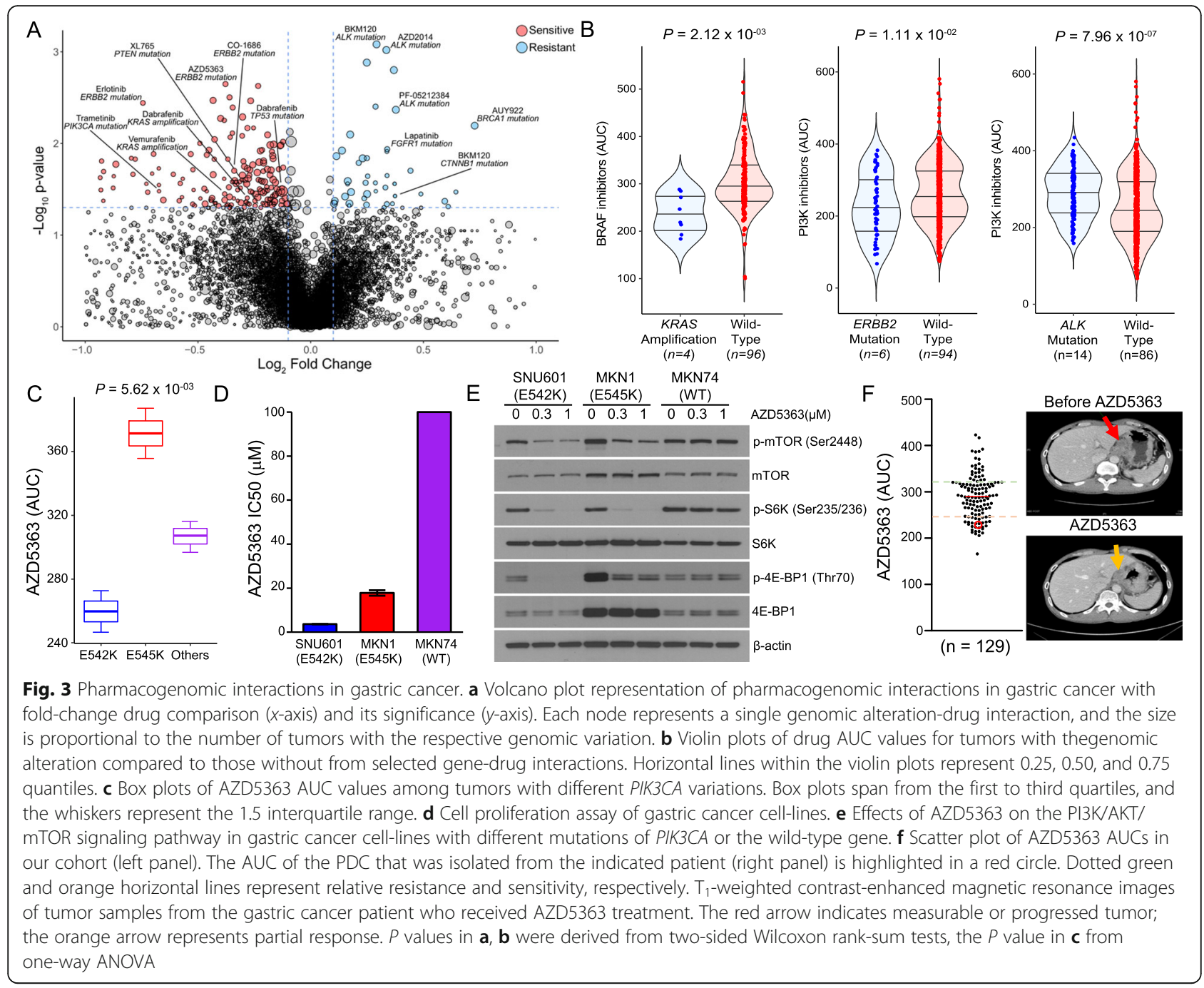

sensitivity profiles of PDC lines against each genomic or molecular aberration (Fig. 3a and Additional file 8: Table S7). Notably, tumors with KRAS amplification were considerably more sensitive to BRAF inhibitors, including vemurafenib and dabrafenib, whereas ERBB2-mutant tumors were therapeutically more susceptible to both EGFR (CO1686 and erlotinib) and PI3K inhibitors (AZD5363, AZD2014, and everolimus). Somatic mutation in FGFR2 conferred increased sensitivity to multi-targeted tyrosine kinase inhibitors, including FGFR- and VEGFR-targeting compounds (Additional file 5: Figure S5). Previous studies have presented molecular rationales for treating $A L K$-mutated tumors with PAM pathway inhibitors [35-37]. However, we discovered that mutation in the ALK receptor tyrosine kinase $(A L K)$ gene was significantly associated with global resistance to a broad range of therapeutics, including those targeting the PI3K-AKT-mTOR (PAM) pathway (e.g., BKM120, AZD2014, PF-05212384) (Fig. 3b). To identify alternative therapeutic avenues for treating $A L K$ mutated tumors, we conducted a genome-wide comparative transcriptome analysis of $A L K$-mutant and $A L K$-wild-type tumors. Interestingly, $L E F 1$ was strongly activated in tumors with $A L K$ mutation, and single sample gene set enrichment analysis (ssGSEA) consistently demonstrated upregulation of the WNT signaling pathway (Additional file 5: Figure S6). Collectively, these results suggest potential therapeutic benefits of WNT-mediated therapy in patients with $A L K$-mutated tumors.

Somatic mutations in PIK3CA, which encodes the catalytic subunit of the phosphatidylinositol 3-kinase (PI3K) complex, have been detected in a broad spectrum of tumor types, including gastric cancer [3, 38-40]. These mutations promote the activation of protooncogenic signaling pathways, rendering cells susceptible to malignant transformation. Furthermore, previous studies have shown that PIK3CA mutation is a key molecular determinant to AKT inhibition response in gastric cancer cell-lines [41]. Although there are multiple variations of PIK3CA mutation, the "hot-spot" mutations are located within the helical (exon 9) or kinase (exon 
20) domains [42]. While previous studies have investigated the potential associations between various PIK3CA mutations and clinical prognosis [43-45], prediction of pharmacological vulnerability based on these mutations remains elusive. Interestingly, when we evaluated drug sensitivities to PI3K compounds in PIK3CA-mutant tumors, we found that SNVs that lead to E542K amino acid substitution induced the most robust susceptibility to an AKT inhibitor (AZD5363) compared to other PIK3CA mutations (Fig. 3c). To functionally validate and explore the AZD5363 and PIK3CA-E542K association and its biological effects, we assessed therapeutic efficacy of AZD5363 in PIK3CA-E542K-mutant, PIK3CAE545K-mutant, and PIK3CA wild-type gastric cell-lines. Consistent with the previous findings in PDC models, cytotoxic activity of AZD5363 was the most significant in PIK3CA-E542K-mutant tumor cells (Fig. 3d and Additional file 5: Figure S7A). Immunoblot analyses of PI3K pathway downstream effectors, including phosphorylation of mTOR, S6K, and 4E-BP1, demonstrated robust inhibition in the presence of AZD5363 treatment (Fig. 3e). Moreover, combinational treatment of AZD5363 with Taxol further increased apoptotic activities (Additional file 5 : Figure S7B). Our findings were further corroborated by clinical data; PDC progeny from a gastric cancer patient with PIK3CA-E542K mutation exhibited profound response to AZD5363, marked by partial clinical response (Fig. 3f). Collectively, these findings suggest the clinical feasibility of patient-derived pharmacogenomics as a potential predictor in patient enrichment trials.

\section{Identification of molecular determinants that dictate drug sensitivity to gefitinib}

A substantial number of studies have demonstrated that EGFR overexpression has been correlated with more malignant phenotypic state and dismal clinical outcomes in gastric cancer patients, suggesting EGFR as a therapeutically exploitable target [46-51]. We have previously demonstrated the clinical feasibility of drug screeningguided precision therapy and initiated a prospective evaluation in gastric cancer patients to examine potential responses to sunitinib, imatinib, and gefitinib [12] (National Clinical Trial [NCT] \#03170180). Therefore, we sought to identify potential molecular or genomic determinants that could aid in design of effective EGFR targeted therapy, specifically utilizing gefitinib, in treating gastric cancer patients. Previous studies have shown that gene expression profiles can be applied to elucidate the biological mechanisms that underlie complex cellular signaling pathways that are associated with drug response [12, 13, 52-55]. Therefore, we employed elasticnet regression model-based analysis using gene expression profiles combined with previously known drug targets, protein-protein interaction networks, and genomic features. As a result, we identified multiple transcriptome molecules that were highly associated with gefitinib response, including RNF11, NTPCR, and RNF220 (Fig. 4a). Among these, the transcriptional expression level of RNF11 demonstrated the most robust correlation (Fig. 4b). Moreover, RNF11 showed direct correlations with other EGFR inhibitors, including AEE788, dacomitinib, and lapatinib as well (Additional file 5: Figure S8). RNF11 encodes ring finger protein 11, and earlier studies have postulated that RNF11 interacts with SARA and ESCRT-0 subunits STAM2 and Eps15b to delay the degradation of EGF-activated EGFR [56]. Interestingly, small interfering RNA (siRNA)-mediated knockdown of RNF11 in two different RNF11 ${ }^{\text {high }}$ gastric cancer cells (SNU5 and NCI-N87) conferred increased sensitivities to gefitinib (Fig. 4c, d and Additional file 5: Figure S9A). Consistently, silencing of RNF11 combined with gefitinib attenuated phosphorylation level of EGFR and its downstream molecule, phospho-AKT, further corroborating that RNF11 has potential as a molecular predictor of intrinsic resistance to EGFR inhibitors (Fig. 4e and Additional file 5: Figure S9B). Overall, our results provide a therapeutically exploitable genomic marker of drug sensitivity that may aid in the design of future biomarker-driven clinical trials in EGFR-targeted therapy.

\section{Discussion}

The fundamental principle of precision oncology is that molecular characterization of the tumor enables optimal patient-tailored therapy $[57,58]$. With the exponential increase in systematic tumor genome sequencing efforts in recent years, molecular aberrations that govern essential cellular programs that are therapeutically exploitable have been identified [34, 59, 60]. However, substantial evidence highlights the current limitations in predicting successful clinical therapies on the sole basis of computational data $[61,62]$. Therefore, systematic evaluation of tumor genome and simultaneous assessment of drug sensitivities have become the next step towards addressing precision oncology therapy. In the present study, we generated a drug sensitivity dataset based on 131 patient-derived tumor specimens that was molecularly annotated to explore dynamic pharmacogenomic interactions in gastric cancers. Consistent with previous genomic characterization of gastric cancers based on molecular classification, we observed enrichments of PIK3CA and TP53 somatic mutations in EBV-positive and HCNA-subtype gastric tumors, respectively, whereas $C D H 1$ and $N F 1$ aberrations were evident in genomically stable (LCNA) tumors. We also identified lineage-specific drug associations, for example, between EGFR inhibitors and signet-ring tumors, and VEGFR compounds and diffuse-type tumors. Consistent herewith, the recent REGARD clinical trial revealed that the VEGFR2 antagonist ramucirumab showed 


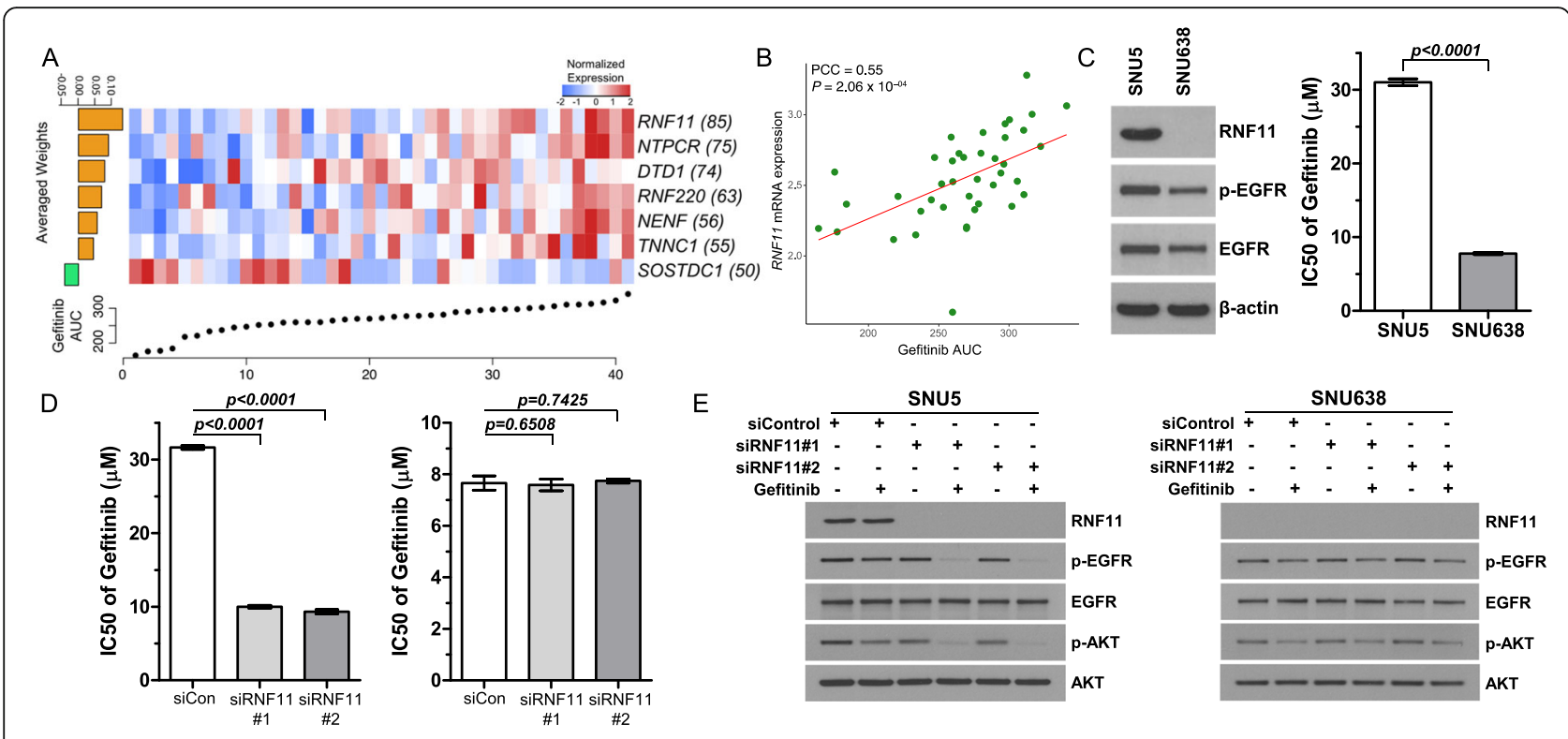

Fig. 4 Transcriptome correlates of gefitinib sensitivity. a Elastic-net regression results of transcriptome features that predict pharmacological response to gefitinib. The bottom scatter plot represents drug response for gefitinib-treated tumors. The upper heatmap shows the top extracted features in the model. The left bar graph shows the averaged weight of each predictive feature. The number of appearances in 100 bootstraps is indicated in parentheses. b Scatter plot revealing a linear correlation between gefitinib AUC and RNF11 transcriptome expression. Correlation coefficients and $P$ values were obtained by Pearson correlation analysis. c Immunoblot analysis of RNF11, p-EGFR, EGFR in gastric cancer cell-lines. $\beta$-Actin was used as a loading control (left panel). Cell proliferation assay in EGFR-activated gastric cancer cell-lines (right panel). Cancer cells were exposed to gefitinib for $72 \mathrm{~h}$, and then, cell viability was measured. d Gastric cancer cell-lines with high (SNU5; left panel) and low (SNU638; right panel) RNF11 expression were transiently transfected with $10 \mathrm{nM}$ of siRNF11 and treated with gefitinib for $72 \mathrm{~h}$ the next day. The results are represented as the mean \pm SD of triplicate wells and are representative of three independent experiments. e Immunoblot analysis of EGFR signaling-related molecules, including p-EGFR, EGFR, p-AKT, and AKT in gastric cancer cell-lines that were transiently transfected with $10 \mathrm{nM}$ of siRNF11 and treated with gefitinib for $4 \mathrm{~h}$ the next day. $P$ values in $\mathbf{c}$, $\mathbf{d}$ were derived from two-sided Student's $t$ tests

prominent clinical benefits in diffuse tumors compared with intestinal-type tumors based on subgroup analysis [20]. Furthermore, we found that tumors with high chromosomal instability (HCNA) were considerably more sensitive to PARP inhibition treatment and harbored higher frequency of BRCA2 mutations. Targeting BRCA1/2-deficient cancers using PARP inhibitors has been the archetype of synthetic lethality based on the inhibition of DNA damage repair (DDR) pathway. The first-in-class PARP inhibitor, olaparib, has been the most extensively studied compound of DDR inhibitors, and the therapeutic landscape of olaparib has been rapidly expanding [63]. Previous phase II clinical trial showed that olaparib with paclitaxel demonstrated greater overall survival benefits in patients with metastatic gastric cancer, specifically those with low ataxia telangiectasia mutated (ATM) expression level [64]. Unfortunately, the subsequent phase III trial failed to attain its primary objective [65]. The current limitation on PARP inhibition therapy necessitated assessment of additional biomarkers to achieve successful clinical outcomes in gastric cancer. In such context, our results suggest that patients with high chromosomal instability and BRCA2 mutation could potentially benefit from olaparib treatment.

Moreover, through large-scale pharmacogenomic analyses, we suggested inhibition of WNT signaling as a therapeutic option for $A L K$-mutant tumors. Furthermore, we found significant therapeutic vulnerabilities of PIK3CA-E542K mutant tumors to AKT inhibition therapy. Of note, we have previously performed the first and largest prospective molecular-guided targeted therapy in patients with gastric cancer, aligned with eight prespecified genomic biomarkers and ten independent biomarker-associated clinical trials (The VIKTORY Umbrella Trial) [66]. Consistent with previous observations, we discovered that patients with PIK3CA-E542K mutant tumors demonstrated the most robust response to AKT inhibitors when compared with patients harboring E545K, E545G, E453K, or other mutant type tumors. Our findings were further experimentally validated where AZD5363 demonstrated potent cytotoxic activities in PIK3CA-E542K-mutant gastric cancer cell-lines, subsequently downregulating PI3K pathway encoding molecules, including phosphorylation of mTOR, S6K, and $4 \mathrm{E}-\mathrm{BP} 1$. These results collectively suggest that the 
current pharmacogenomic atlas of PDC models support the clinical feasibility of molecular-guided targeted therapy in hopes of expediting personalized treatment.

Lastly, we identified molecular determinants, including $R N F 11$, of the therapeutic response to gefitinib treatment. RNF11 has been presented as a proto-oncogene, delaying the degradation of EGF-activated EGFR signaling pathway components. Previous phase II and III clinical trials using EGFR-targeted agents (cetuximab, panitumumab, nimotuzumab, gefitinib) in gastric cancer were terminated due to insignificant survival advantage [49, 67-69]. However, recent reports suggested the clinical feasibility of EGFRmediated therapy, especially for patients with EGFR amplification in gastric tumors, and revealed potential mechanisms underlying EGFR therapeutic resistance [70]. Notably, we discovered that signet-ring cell-type tumors demonstrated considerable sensitivities to EGFR inhibitors, while tubular types were widely resistant. Conversely, siRNA-mediated knockdown of RNF11 sensitized tumor cells, including tubular-type tumors to gefitinib. These results suggest a potential combinational strategy to circumvent EGFR-mediated therapeutic resistance.

In conclusion, integration of tumor genome and drug sensitivity data is the next step towards precision oncology therapy. As molecular target agents, including trastuzumab and ramucirumab, are currently being used in combination with cytotoxic chemotherapeutic compounds (5FU/CDDP and paclitaxel, respectively) to treat gastric cancer patients [18, 19], we believe that the results in this study provide opportunities to design effective clinical trials and combinational therapeutic strategies in hopes of facilitating clinical application towards personalized treatment in gastric cancer.

\section{Conclusions}

In summary, we have established a systematic framework for genetic prediction of anticancer drug response using patient-derived resources that are molecularly, clinically, and pharmacologically annotated. Through comprehensive pharmacogenomic analyses, we identified lineage-specific drug sensitivities and gene-drug interactions that are well represented within clinical context, including VEGFR inhibitors with diffuse-type tumors and PIK3CA-E542K mutation with AKT inhibition therapy. Furthermore, we suggested RNF11 expression as a predictive biomarker for gefitinib treatment in gastric cancer.

\section{Supplementary information}

Supplementary information accompanies this paper at https://doi.org/10. 1186/s13073-020-0717-8.

Additional file 1: Table S1. List of 60 drugs that were used in the drug sensitivity analysis. Drugs have been annotated based on their chemical and/or generic name, target molecule and clinical phase.
Additional file 2: Table S2. Clinical annotations of 131 gastric cancers used in the drug screening and/or genomic characterization.

Additional file 3: Table S3. List of major cancer-driver genes and sequencing library information for CancerSCAN.

Additional file 4: Table S4. Mutational landscape of gastric cancer. Additional file 5: Figure S1. Unsupervised clustering of gastric cancers. Figure S2. Frequency of major gastric cancer-driver gene alterations. Figure S3. Pharmacological landscape of gastric cancers for 60 molecular-targeted compounds. Figure S4. Subgroup-specific drug sensitivity among gastric cancers. Figure S5. Gene-drug associations among gastric cancers. Figure S6. Pathway enrichment analysis between $A L K$-mutant and ALK wild-type tumors. Figure S7. Pharmacological effects of PIK3CA mutations on AZD5363 and Taxol combination treatment. Figure S8. Correlations between RNF11 mRNA expression level and EGFR inhibitors. Figure S9. siRNA-mediated knockdown of RNF11 promotes therapeutic sensitivity to gefitinib.

Additional file 6: Table S5. Area Under the Curve (AUC) values for 60 drugs in 129 PDCs.

Additional file 7: Table S6. Tumor type-specific drug associations. Wilcoxon rank-sum test was applied to determine the relative difference of drug sensitivity between individual tumor type and the rest.

Additional file 8: Table S7. Pharmacogenomic interactions using integrative analysis of drug sensitivity results (AUC) and genomic alterations. The statistical significance was calculated using Wilcoxon rank-sum test.

\section{Acknowledgements}

We thank the Samsung Medical Center BioBank for providing the biospecimens that were used in this study.

\section{Authors' contributions}

JKS analyzed the therapeutic landscape and pharmacogenomic interactions. JYH provided clinical insights and interpreted the clinical data. I-KL, J-SK, M-HS, and HJK performed cancer cell-line experiments and analyzed the data. JYA, TSS, JHL, JMB, SK, STK, SHP, JOP, YSP, HYL, and WKK contributed to the collection of patient samples and clinical information. K-MK conducted the pathology assessment. $\mathrm{N}-\mathrm{GH}, \mathrm{YL}$, and $\mathrm{MK}$ organized and analyzed the drug-screening experiments. JKS, HJC, and HK processed and analyzed the genomic and transcriptomic data. YJS, MK, YJS, and JYK processed and generated the patientderived cell library. JKS, JYH, MGC, D-HN, and JL wrote the manuscript and analyzed the overall data. MGC, D-HN, and JL designed and supervised the entire project. All authors read and approved the final manuscript.

\section{Funding}

This research was supported by a grant of the Korea Health Technology Research and Development project through the Korea Health Industry Development Institute (KHIDI), funded by the Ministry of Health and Welfare, Republic of Korea (H114C3418), and Korea University Grant (K1923771 and K1925031).

\section{Availability of data and materials}

All newly sequenced data have been deposited in the European Genomephenome Archive (EGA) under accession EGAS00001004106 [71].

\section{Ethics approval and consent to participate}

The study was approved by the committee of Samsung Medical Center (SMC), Seoul, Republic of Korea (IRB file \#201004004), on the use of human samples for experimental studies. Written informed consent was obtained from all study participants prior to enrollment. The research conformed to the principles of the Helsinki Declaration.

\section{Consent for publication}

Written informed consent was obtained from all study participants prior to enrollment.

\section{Competing interests}

Do-Hyun Nam is the CEO of AimedBio Inc. and owns shares of AimedBio Inc. which owns IPs for Avatascan. The remaining authors declare that they have no competing interests. 


\section{Author details}

'Department of Biomedical Sciences, Korea University College of Medicine, Seoul, Republic of Korea. ${ }^{2}$ Division of Hematology-Oncology, Department of Medicine, Samsung Medical Center, Sungkyunkwan University School of Medicine, Seoul, Republic of Korea. ${ }^{3}$ Department of Surgery, Samsung Medical Center, Sungkyunkwan University School of Medicine, Seoul, Republic of Korea. ${ }^{4}$ Department of Pathology, Samsung Medical Center, Sungkyunkwan University School of Medicine, Seoul, Republic of Korea. ${ }^{5}$ Institute for Refractory Cancer Research, Samsung Medical Center, Seoul, Republic of Korea. ${ }^{6}$ Department of Health Sciences and Technology, Samsung Advanced Institute for Health Science and Technology, Sungkyunkwan University, Seoul, Republic of Korea. ${ }^{7}$ Department of Neurosurgery, Samsung Medical Center, Sungkyunkwan University School of Medicine, Seoul, Republic of Korea.

Received: 5 August 2019 Accepted: 31 January 2020

Published online: 18 February 2020

\section{References}

1. Corces MR, Granja JM, Shams S, Louie BH, Seoane JA, Zhou W, Silva TC, Groeneveld C, Wong CK, Cho SW, et al. The chromatin accessibility landscape of primary human cancers. Science. 2018:362(6413).

2. Bailey MH, Tokheim C, Porta-Pardo E, Sengupta S, Bertrand D, Weerasinghe A, Colaprico A, Wendl MC, Kim J, Reardon B, et al, Comprehensive characterization of cancer driver genes and mutations. Cell. 2018:173:371-85 e318.

3. Cancer Genome Atlas Research N. Comprehensive molecular characterization of gastric adenocarcinoma. Nature. 2014;513:202-9.

4. Cancer Genome Atlas Research N, Weinstein JN, Collisson EA, Mills GB, Shaw KR, Ozenberger BA, Ellrott K, Shmulevich I, Sander C, Stuart JM. The cancer genome atlas pan-cancer analysis project. Nat Genet. 2013:45:1113-20.

5. Cancer Genome Atlas Research N. Comprehensive genomic characterization defines human glioblastoma genes and core pathways. Nature. 2008;455: 1061-8.

6. Cancer Genome Atlas Research N. Integrated genomic analyses of ovarian carcinoma. Nature. 2011;474:609-15.

7. Nik-Zainal S, Davies H, Staaf J, Ramakrishna M, Glodzik D, Zou X Martincorena I, Alexandrov LB, Martin S, Wedge DC, et al. Landscape of somatic mutations in 560 breast cancer whole-genome sequences. Nature. 2016:534:47-54.

8. Barretina J, Caponigro G, Stransky N, Venkatesan K, Margolin AA, Kim S, Wilson CJ, Lehar J, Kryukov GV, Sonkin D, et al. The cancer cell line encyclopedia enables predictive modelling of anticancer drug sensitivity. Nature. 2012:483:603-7.

9. Garnett MJ, Edelman EJ, Heidorn SJ, Greenman CD, Dastur A, Lau KW, Greninger P, Thompson IR, Luo X, Soares J, et al. Systematic identification of genomic markers of drug sensitivity in cancer cells. Nature. 2012;483:570-5.

10. Iorio F, Knijnenburg TA, Vis DJ, Bignell GR, Menden MP, Schubert M, Aben N, Goncalves E, Barthorpe S, Lightfoot H, et al. A landscape of pharmacogenomic interactions in cancer. Cell. 2016;166:740-54.

11. Wang Y, Wang Z, Xu J, Li J, Li S, Zhang M, Yang D. Systematic identification of non-coding pharmacogenomic landscape in cancer. Nat Commun. 2018; 9:3192.

12. Lee JK, Liu Z, Sa JK, Shin S, Wang J, Bordyuh M, Cho HJ, Elliott O, Chu T, Choi SW, et al. Pharmacogenomic landscape of patient-derived tumor cells informs precision oncology therapy. Nat Genet. 2018;50:1399-411.

13. Sa JK, Hwang JR, Cho YJ, Ryu JY, Choi JJ, Jeong SY, Kim J, Kim MS, Paik ES, Lee YY, et al. Pharmacogenomic analysis of patient-derived tumor cells in gynecologic cancers. Genome Biol. 2019;20:253.

14. Crew KD, Neugut Al. Epidemiology of gastric cancer. World J Gastroenterol. 2006:12:354-62

15. Uemura N, Okamoto S, Yamamoto S, Matsumura N, Yamaguchi S, Yamakido M, Taniyama K, Sasaki N, Schlemper RJ. Helicobacter pylori infection and the development of gastric cancer. N Engl J Med. 2001;345:784-9.

16. Ajani JA, Lee J, Sano T, Janjigian YY, Fan D, Song S. Gastric adenocarcinoma. Nat Rev Dis Primers. 2017:3:17036.

17. Hu B, El Hajj N, Sittler S, Lammert N, Barnes R, Meloni-Ehrig A. Gastric cancer: classification, histology and application of molecular pathology. J Gastrointest Oncol. 2012;3:251-61.

18. Bang YJ, Van Cutsem E, Feyereislova A, Chung HC, Shen L, Sawaki A, Lordick F, Ohtsu A, Omuro Y, Satoh T, et al. Trastuzumab in combination with chemotherapy versus chemotherapy alone for treatment of HER2-positive advanced gastric or gastro-oesophageal junction cancer (ToGA): a phase 3, open-label, randomised controlled trial. Lancet. 2010;376:687-97.

19. Wilke H, Muro K, Van Cutsem E, Oh SC, Bodoky G, Shimada Y, Hironaka S, Sugimoto N, Lipatov O, Kim TY, et al. Ramucirumab plus paclitaxel versus placebo plus paclitaxel in patients with previously treated advanced gastric or gastro-oesophageal junction adenocarcinoma (RAINBOW): a doubleblind, randomised phase 3 trial. Lancet Oncol. 2014;15:1224-35.

20. Fuchs CS, Tomasek J, Yong CJ, Dumitru F, Passalacqua R, Goswami C, Safran H, Dos Santos LV, Aprile G, Ferry DR, et al. Ramucirumab monotherapy for previously treated advanced gastric or gastro-oesophageal junction adenocarcinoma (REGARD): an international, randomised, multicentre, placebo-controlled, phase 3 trial. Lancet. 2014;383:31-9.

21. Zhang JH, Chung TD, Oldenburg KR. A simple statistical parameter for use in evaluation and validation of high throughput screening assays. J Biomol Screen. 1999:4:67-73.

22. Liu $Y$, Sethi NS, Hinoue $T$, Schneider BG, Cherniack AD, Sanchez-Vega F, Seoane JA, Farshidfar F, Bowlby R, Islam M, et al. Comparative molecular analysis of gastrointestinal adenocarcinomas. Cancer Cell. 2018;33:721-35 e728.

23. Yang $M$, Huang $C Z$. Mitogen-activated protein kinase signaling pathway and invasion and metastasis of gastric cancer. World J Gastroenterol. 2015;21: 11673-9.

24. Chen K, Yang D, Li X, Sun B, Song F, Cao W, Brat DJ, Gao Z, Li H, Liang H, et al. Mutational landscape of gastric adenocarcinoma in Chinese: implications for prognosis and therapy. Proc Natl Acad Sci U S A. 2015;112:1107-12.

25. Wang K, Yuen ST, Xu J, Lee SP, Yan HH, Shi ST, Siu HC, Deng S, Chu KM, Law $\mathrm{S}$, et al. Whole-genome sequencing and comprehensive molecular profiling identify new driver mutations in gastric cancer. Nat Genet. 2014;46: $573-82$.

26. Shin YJ, Sa JK, Lee Y, Kim D, Chang N, Cho HJ, Son M, Oh MYT, Shin K, Lee JK, et al. PIP4K2A as a negative regulator of PI3K in PTEN-deficient glioblastoma. J Exp Med. 2019;216:1120-34.

27. Lee JY, Kim SY, Park C, Kim NK, Jang J, Park K, Yi JH, Hong M, Ahn T, Rath O, et al. Patient-derived cell models as preclinical tools for genome-directed targeted therapy. Oncotarget. 2015;6:25619-30.

28. Lee J, Kotliarova S, Kotliarov Y, Li A, Su Q, Donin NM, Pastorino S, Purow BW Christopher $\mathrm{N}$, Zhang $\mathrm{W}$, et al. Tumor stem cells derived from glioblastomas cultured in bFGF and EGF more closely mirror the phenotype and genotype of primary tumors than do serum-cultured cell lines. Cancer Cell. 2006;9: 391-403.

29. Moore K, Colombo N, Scambia G, Kim BG, Oaknin A, Friedlander M, Lisyanskaya A, Floquet A, Leary A, Sonke GS, et al. Maintenance olaparib in patients with newly diagnosed advanced ovarian cancer. N Engl I Med. 2018:379:2495-505.

30. Robson M, Im SA, Senkus E, Xu B, Domchek SM, Masuda N, Delaloge S, Li W. Tung $N$, Armstrong $A$, et al. Olaparib for metastatic breast cancer in patients with a germline BRCA mutation. N Engl J Med. 2017;377:523-33.

31. Hamburg MA, Collins FS. The path to personalized medicine. N Engl J Med. 2010;363:301-4.

32. Chapman PB, Hauschild A, Robert C, Haanen JB, Ascierto P, Larkin J, Dummer R, Garbe C, Testori A, Maio M, et al. Improved survival with vemurafenib in melanoma with BRAF V600E mutation. N Engl J Med. 2011; 364:2507-16.

33. Slamon DJ, Leyland-Jones B, Shak S, Fuchs H, Paton V, Bajamonde A, Fleming T, Eiermann W, Wolter J, Pegram M, et al. Use of chemotherapy plus a monoclonal antibody against HER2 for metastatic breast cancer that overexpresses HER2. N Engl J Med. 2001;344:783-92.

34. Tiacci E, Trifonov V, Schiavoni G, Holmes A, Kern W, Martelli MP, Pucciarini A, Bigerna B, Pacini R, Wells VA, et al. BRAF mutations in hairy-cell leukemia. N Engl J Med. 2011;364:2305-15.

35. Hallberg B, Palmer RH. The role of the ALK receptor in cancer biology. Ann Oncol. 2016;27(Suppl 3):iii4-iii15.

36. Holla VR, Elamin YY, Bailey AM, Johnson AM, Litzenburger BC, Khotskaya YB, Sanchez NS, Zeng J, Shufean MA, Shaw KR, et al. ALK: a tyrosine kinase target for cancer therapy. Cold Spring Harb Mol Case Stud. 2017; 3:a001115.

37. Moore NF, Azarova AM, Bhatnagar N, Ross KN, Drake LE, Frumm S, Liu QS, Christie AL, Sanda T, Chesler L, et al. Molecular rationale for the use of PIBK AKT/mTOR pathway inhibitors in combination with crizotinib in ALKmutated neuroblastoma. Oncotarget. 2014;5:8737-49. 
38. Harada K, Baba Y, Shigaki H, Ishimoto T, Miyake K, Kosumi K, Tokunaga R, Izumi D, Ohuchi M, Nakamura K, et al. Prognostic and clinical impact of PIK3CA mutation in gastric cancer: pyrosequencing technology and literature review. BMC Cancer. 2016;16:400.

39. Samuels $Y$, Waldman T. Oncogenic mutations of PIK3CA in human cancers Curr Top Microbiol Immunol. 2010;347:21-41.

40. Karakas B, Bachman KE, Park BH. Mutation of the PIK3CA oncogene in human cancers. Br J Cancer. 2006;94:455-9.

41. Li J, Davies BR, Han S, Zhou M, Bai Y, Zhang J, Xu Y, Tang L, Wang H, Liu YJ, et al. The AKT inhibitor AZD5363 is selectively active in PI3KCA mutant gastric cancer, and sensitizes a patient-derived gastric cancer xenograft model with PTEN loss to Taxotere. J Transl Med. 2013;11:241.

42. Samuels Y, Diaz LA Jr, Schmidt-Kittler O, Cummins JM, Delong L, Cheong I, Rago C, Huso DL, Lengauer C, Kinzler KW, et al. Mutant PIK3CA promotes cell growth and invasion of human cancer cells. Cancer Cell. 2005;7:561-73.

43. Mjos S, Werner HMJ, Birkeland E, Holst F, Berg A, Halle MK, Tangen IL, Kusonmano K, Mauland KK, Oyan AM, et al. PIK3CA exon9 mutations associate with reduced survival, and are highly concordant between matching primary tumors and metastases in endometrial cancer. Sci Rep. 2017;7:10240.

44. Shigaki $H$, Baba $Y$, Watanabe M, Murata A, Ishimoto T, Iwatsuki M, Iwagami S, Nosho K, Baba H. PIK3CA mutation is associated with a favorable prognosis among patients with curatively resected esophageal squamous cell carcinoma. Clin Cancer Res. 2013;19:2451-9.

45. Dumont AG, Dumont SN, Trent JC. The favorable impact of PIK3CA mutations on survival: an analysis of 2587 patients with breast cancer. Chin J Cancer. 2012:31:327-34.

46. Kanat O, O'Neil B, Shahda S. Targeted therapy for advanced gastric cancer: a review of current status and future prospects. World J Gastrointest Oncol. 2015:7:401-10

47. Salomon DS, Brandt R, Ciardiello F, Normanno N. Epidermal growth factorrelated peptides and their receptors in human malignancies. Crit Rev Oncol Hematol. 1995;19:183-232.

48. Nicholson RI, Gee JM, Harper ME. EGFR and cancer prognosis. Eur J Cancer. 2001;37(Suppl 4):S9-15.

49. Rojo F, Tabernero J, Albanell J, Van Cutsem E, Ohtsu A, Doi T, Koizumi W, Shirao K, Takiuchi H, Ramon y Cajal S, Baselga J. Pharmacodynamic studies of gefitinib in tumor biopsy specimens from patients with advanced gastric carcinoma. J Clin Oncol. 2006;24:4309-16.

50. Adelstein DJ, Rodriguez CP, Rybicki LA, Ives DI, Rice TW. A phase II trial of gefitinib for recurrent or metastatic cancer of the esophagus or gastroesophageal junction. Investig New Drugs. 2012;30:1684-9.

51. Kim MA, Lee HS, Lee HE, Jeon YK, Yang HK, Kim WH. EGFR in gastric carcinomas: prognostic significance of protein overexpression and high gene copy number. Histopathology. 2008;52:738-46.

52. DeRisi JL, lyer VR, Brown PO. Exploring the metabolic and genetic control of gene expression on a genomic scale. Science. 1997;278:680-6.

53. Lamb J, Crawford ED, Peck D, Modell JW, Blat IC, Wrobel MJ, Lerner J, Brunet JP, Subramanian A, Ross KN, et al. The Connectivity Map: using gene-expression signatures to connect small molecules, genes, and disease. Science. 2006:313:1929-35.

54. Lamb J, Ramaswamy S, Ford HL, Contreras B, Martinez RV, Kittrell FS, Zahnow CA, Patterson N, Golub TR, Ewen ME. A mechanism of cyclin D1 action encoded in the patterns of gene expression in human cancer. Cell. 2003:114:323-34

55. Mulligan G, Mitsiades C, Bryant B, Zhan F, Chng WJ, Roels S, Koenig E, Fergus A, Huang $Y$, Richardson $P$, et al. Gene expression profiling and correlation with outcome in clinical trials of the proteasome inhibitor bortezomib. Blood. 2007;109:3177-88.

56. Kostaras E, Sflomos G, Pedersen NM, Stenmark H, Fotsis T, Murphy C. SARA and RNF11 interact with each other and ESCRT-0 core proteins and regulate degradative EGFR trafficking. Oncogene. 2013;32:5220-32.

57. O'Brien SG, Guilhot F, Larson RA, Gathmann I, Baccarani M, Cervantes F, Cornelissen JJ, Fischer T, Hochhaus A, Hughes T, et al. Imatinib compared with interferon and low-dose cytarabine for newly diagnosed chronic-phase chronic myeloid leukemia. N Engl J Med. 2003;348:994-1004.

58. Loeb LA. Human cancers express mutator phenotypes: origin consequences and targeting. Nat Rev Cancer. 2011;11:450-7.

59. Brennan CW, Verhaak RG, McKenna A, Campos B, Noushmehr H, Salama SR, Zheng S, Chakravarty D, Sanborn JZ, Berman SH, et al. The somatic genomic landscape of glioblastoma. Cell. 2013;155:462-77.
60. Gao Q, Liang WW, Foltz SM, Mutharasu G, Jayasinghe RG, Cao S, Liao WW, Reynolds SM, Wyczalkowski MA, Yao L, et al. Driver fusions and their implications in the development and treatment of human cancers. Cell Rep. 2018:23:227-38 e223.

61. Le Tourneau C, Delord JP, Goncalves A, Gavoille C, Dubot C, Isambert N, Campone M, Tredan O, Massiani MA, Mauborgne C, et al. Molecularly targeted therapy based on tumour molecular profiling versus conventional therapy for advanced cancer (SHIVA): a multicentre, open-label, proof-of-concept, randomised, controlled phase 2 trial. Lancet Oncol. 2015;16:1324-34.

62. Di Nicolantonio F, Arena S, Tabernero J, Grosso S, Molinari F, Macarulla T, Russo M, Cancelliere C, Zecchin D, Mazzucchelli L, et al. Deregulation of the PI3K and KRAS signaling pathways in human cancer cells determines their response to everolimus. J Clin Invest. 2010;120:2858-66.

63. Pilie PG, Tang C, Mills GB, Yap TA. State-of-the-art strategies for targeting the DNA damage response in cancer. Nat Rev Clin Oncol. 2019;16:81-104.

64. Bang YJ, Im SA, Lee KW, Cho JY, Song EK, Lee KH, Kim YH, Park JO, Chun HG, Zang DY, et al. Randomized, double-blind phase II trial with prospective classification by ATM protein level to evaluate the efficacy and tolerability of olaparib plus paclitaxel in patients with recurrent or metastatic gastric cancer. J Clin Oncol. 2015;33:3858-65.

65. Bang YJ, Xu RH, Chin K, Lee KW, Park SH, Rha SY, Shen L, Qin S, Xu N, Im SA et al. Olaparib in combination with paclitaxel in patients with advanced gastric cancer who have progressed following first-line therapy (GOLD): a double-blind, randomised, placebo-controlled, phase 3 trial. Lancet Oncol. 2017;18:1637-51.

66. Lee J, Kim ST, Kim K, Lee H, Kozarewa I, Mortimer PGS, Odegaard JI, Harrington EA, Lee J, Lee T, et al. Tumor genomic profiling guides patients with metastatic gastric cancer to targeted treatment: the VIKTORY Umbrella Trial. Cancer Discov. 2019;9:1388-405.

67. Lordick F, Kang YK, Chung HC, Salman P, Oh SC, Bodoky G, Kurteva G, Volovat C, Moiseyenko VM, Gorbunova V, et al. Capecitabine and cisplatin with or without cetuximab for patients with previously untreated advanced gastric cancer (EXPAND): a randomised, open-label phase 3 trial. Lancet Oncol. 2013;14:490-9.

68. Waddell T, Chau I, Cunningham D, Gonzalez D, Okines AF, Okines C, Wotherspoon A, Saffery C, Middleton G, Wadsley J, et al. Epirubicin, oxaliplatin, and capecitabine with or without panitumumab for patients with previously untreated advanced oesophagogastric cancer (REAL3): a randomised, open-label phase 3 trial. Lancet Oncol. 2013;14:481-9.

69. Satoh T, Lee KH, Rha SY, Sasaki Y, Park SH, Komatsu Y, Yasui H, Kim TY, Yamaguchi K, Fuse N, et al. Randomized phase II trial of nimotuzumab plus irinotecan versus irinotecan alone as second-line therapy for patients with advanced gastric cancer. Gastric Cancer. 2015;18:824-32.

70. Maron SB, Alpert L, Kwak HA, Lomnicki S, Chase L, Xu D, O'Day E, Nagy RJ, Lanman RB, Cecchi F, et al. Targeted therapies for targeted populations: anti-EGFR treatment for EGFR-amplified gastroesophageal adenocarcinoma. Cancer Discov. 2018;8:696-713.

71. Sa JK, Hong JY, Lee IK, Kim JS, Sim MH, Kim HJ, An JY, Sohn TS, Lee JH, Bae $\mathrm{JM}$, et al. Comprehensive pharmacogenomic characterization of gastric cancer. Eur Genome-phenome Arch. 2019. https://www.ebi.ac.uk/ega/ studies/EGAS00001004106. Accessed 16 Feb 2020

\section{Publisher's Note}

Springer Nature remains neutral with regard to jurisdictional claims in published maps and institutional affiliations.

Ready to submit your research? Choose BMC and benefit from:

- fast, convenient online submission

- thorough peer review by experienced researchers in your field

- rapid publication on acceptance

- support for research data, including large and complex data types

- gold Open Access which fosters wider collaboration and increased citations

- maximum visibility for your research: over $100 \mathrm{M}$ website views per year

At $\mathrm{BMC}$, research is always in progress.

Learn more biomedcentral.com/submissions 